\title{
Blockade of 146b-5p promotes inflammation in atherosclerosis-associated foam cell formation by targeting TRAF6
}

\author{
NAN LIN ${ }^{1,2}$ and YI AN $^{3}$ \\ ${ }^{1}$ Department of Clinical Medicine, Medical College, Qingdao University, Qingdao, Shandong 266021; \\ ${ }^{2}$ Division of Cardiology, The Affiliated Hospital of Jining Medical University, Jining, Shandong 272100; \\ ${ }^{3}$ Division of Cardiology, The Affiliated Hospital of Qingdao University, Qingdao, Shandong 266100, P.R. China
}

Received November 9, 2016; Accepted June 22, 2017

DOI: $10.3892 / \mathrm{etm} .2017 .5121$

\begin{abstract}
Atherosclerosis (AS) is a chronic inflammation in response to lipid accumulation. Increasing evidence has demonstrated that numerous microRNAs (miRs) have critical roles in inflammatory responses. A previous study suggested that miR-146b-5p is possibly associated with AS; however, its exact role has remained largely elusive. The present study aimed to investigate the potential role of miR-146b-5p in AS and to explore the underlying mechanism. Fist, the levels of miR-146b-5p were determined in foam cells and clinical specimens from patients with AS by reverse-transcription quantitative PCR. The role of miR-146b-5p in AS was then investigated by using miR-146b-5p inhibitor. The results demonstrated that the expression levels of miR-146b-5p were elevated in the lesions of patients with AS. In addition, the levels of miR-146b-5p in THP-1 cells stimulated with phorbol 12-myristate 13 -acetate $(100 \mathrm{nM})$ to induce their differentiation into macrophages were dose- and time-dependently elevated by oxidized low-density lipoprotein treatment applied for inducing foam cell formation. miR-146b-5p was also revealed to directly target tumor necrosis factor receptor-associated factor 6 (TRAF6), which functions as a signal transducer in the nuclear factor- $\kappa \mathrm{B}(\mathrm{NF}-\kappa \mathrm{B})$ pathway. Furthermore, the present study reported for the first time that miR-146b-5p inhibition promotes the inflammatory response and enhances lipid uptake during foam cell formation. In conclusion, miR-146b-5p inhibition promoted chronic inflammation and had a detrimental role during AS-associated foam cell formation by targeting TRAF6.
\end{abstract}

Correspondence to: Dr Yi An, Division of Cardiology, The Affiliated Hospital of Qingdao University, 59 Hai'er Road, Qingdao, Shandong 266100, P.R. China

E-mail: any_medical@163.com

Key words: atherosclerosis, microRNA-146b-5p, tumor necrosis factor receptor-associated factor 6 , chronic inflammation

\section{Introduction}

Atherosclerosis (AS), one of the most common cardiovascular diseases, is a major cause of morbidity and mortality worldwide (1). Hyperlipidemia, monocyte recruitment, differentiation into macrophages, foam cell formation and induced inflammation are the key cellular events of AS (2). Chronic inflammation has a key role in the occurrence and development of AS (3), and macrophages with the ability to stimulate the vascular inflammatory reaction are the major effector cells throughout the pathological process of AS (4). Therefore, genes and cytokines involved in immune system control are essential in regulating AS. Recent studies have indicated the potential roles of microRNAs (miRNAs) in the regulation of AS-associated processes $(5,6)$.

miRNAs are small ( 22 nucleotides in length), endogenous, non-coding, single-stranded RNAs, which have important roles in gene expression regulation by binding to the 3'-untranslated region (UTR) of target mRNAs $(7,8)$. A growing body of evidence suggested that miRNAs have different roles in the development of AS. Li et al (9) reported that increased miR-155 relieves chronic inflammation in AS. Xu et al (10) suggested that miR-135b-5p and miR-499a-3p promoted cell proliferation and migration in AS. Zhang et al (11) demonstrated that miR-26a prevented endothelial cell apoptosis under AS conditions. Ouimet et al (12) found that miR-33 antagonism exerted atheroprotective roles via regulating macrophage-associated inflammation.

A previous study demonstrated upregulation of miR-146b-5p in oxidized low-density lipoprotein (oxLDL)stimulated monocytes (13); however, to the best of our knowledge, no further study on the role of miR-146b-5p in AS has been performed. Thus, the present study investigated the potential role of miR-146b-5p in AS and explored the underlying molecular mechanisms.

\section{Materials and methods}

Specimens. A total of 10 pairs of atherosclerotic lesion tissues and normal veins were identified and collected during biopsies from 10 patients (gender ratio: 1:1; age, ranging from 42 to 59 years old) with AS who were diagnosed by clinical symptoms and angiography at the Affiliated Hospital of Qingdao 
University (Qingdao, China) between August 2015 and August 2016. The exclusion criteria of the patients were as previously described (9). Informed consent was obtained from each patient. The present study was approved by the Affiliated Hospital of Qingdao University (Qingdao, China).

Cell culture and foam cell model construction. The THP-1 human monocytic and the HEK293T human embryonic kidney cell line were obtained from the American Type Culture Collection (Manassas, VA, USA). THP-1 cells were grown in RPMI-1640 medium, supplemented with $10 \%$ fetal bovine serum (both from Gibco; Thermo Fisher Scientific, Inc., Waltham, MA USA), and $1 \%$ streptomycin and penicillin mixed solution (Thermo Fisher Scientific, Inc.). HEK-293T cells were cultured in Dulbecco's modified Eagle's medium (Gibco; Thermo Fisher Scientific, Inc.). All cells were incubated in a humidified atmosphere with $5 \% \mathrm{CO}_{2}$ at $37^{\circ} \mathrm{C}$. A foam cell model was established as previously described (14-16). In brief, THP-1 cells were first seeded in culture plates at $1 \times 10^{6}$ cells $/ \mathrm{ml}$ with $100 \mathrm{nM}$ phorbol 12-myristate 13-acetate (PMA; Sangon Biotech Co., Ltd., Shanghai, China) for $12 \mathrm{~h}$ to differentiate them into macrophages. Subsequently, the cells were stimulated with different concentrations of oxLDL $(10,50$ or $100 \mu \mathrm{g} / \mathrm{ml}$; Sangon Biotech Co., Ltd.) for specific durations $(0,6$ or $12 \mathrm{~h})$ in order to induce foam cell formation; control cells were treated with PBS.

Oil Red $O$ staining. Macrophages derived from THP-1 cells were transfected with a miR-146b-5p inhibitor or its negative control (GenScript Biotech Corporation; Piscataway, NJ, USA) using Lipofectamine 2000 transfection reagent (Invitrogen; Thermo Fisher Scientific, Inc.). Following transfection with miR-146b-5p inhibitor or control, cells were treated with oxidized low-density lipoprotein $(50 \mu \mathrm{g} / \mathrm{ml})$ for $24 \mathrm{~h}$. The cells were then washed with PBS, fixed with $4 \%$ paraformaldehyde, stained with Oil Red O (Sangon Biotech Co., Ltd.) at room temperature for $20 \mathrm{~min}$, and then de-stained with $60 \%$ isopropanol for $1 \mathrm{~min}$. The foam cells were then imaged by using a microscope (Olympus Corporation,, Tokyo, Japan) at a magnification of $\mathrm{x} 40$.

RNA isolation and reverse-transcription quantitative polymerase chain reaction $(R T-q P C R)$. miR-146b-5p was extracted from atherosclerotic lesion tissues and normal veins by using the mirVana PARIS kit (cat no. AM1556; Ambion; Thermo Fisher Scientific, Inc.) in line with the manufacturer's instructions. The TaqMan MicroRNA Reverse Transcription kit (cat no. 4366596; Applied Biosystems; Thermo Fisher Scientific, Inc.) was used for RT of miRNA, and the complementary (c)DNA was amplified by PCR using TaqMan Fast Advanced Master Mix (cat no. 4444556; Applied Biosystems; Thermo Fisher Scientific, Inc.) according to the manufacturer's instructions. The amplification conditions were as follows: 37 cycles of denaturation at $95^{\circ} \mathrm{C}$ for $10 \mathrm{sec}$, followed by annealing and extension at $58^{\circ} \mathrm{C}$ for $60 \mathrm{sec}$. U6 was used as an endogenous control of miRNA expression. The primer sequences were as follows: miR-146b-5p forward, 5'-TGA CCCATCCTGGGCCTCAA-3' and reverse, 5'-CCAGTGGGC AAGATGTGGGCC-3'; and U6 forward, 5'GCTTCGGCA
GCACATATACTAAAAT3' and reverse, 5'CGCTTCACG AATTTGCGTGTCAT3'.

For tumor necrosis factor (TNF) receptor-associated factor 6 (TRAF6) mRNA expression analysis, total RNA from THP-1-derived macrophages was isolated by using TRIzol reagent (Invitrogen; Thermo Fisher Scientific, Inc.). cDNA was obtained by using a RT-PCR detection kit (cat no. 18091050; Invitrogen; Thermo Fisher Scientific, Inc.) following the manufacturer's instructions. Real-time PCR was performed to amplify the synthesized cDNA by using the Fast SYBR Green Master Mix (cat no. 4385610; Applied Biosystems; Thermo Fisher Scientific, Inc.) according to the manufacturer's instructions. Amplification conditions were $95^{\circ} \mathrm{C}$ for $10 \mathrm{~min}$, followed by 38 cycles at $95^{\circ} \mathrm{C}$ for $15 \mathrm{sec}$, and $72^{\circ} \mathrm{C}$ for $30 \mathrm{sec}$. GAPDH acted as an internal control. The $2^{-\Delta \Delta C a}$ method was used to calculate the relative gene expression (17). The primer sequences were as follows: TRAF6 forward, 5'-GAGTTTGAC CCACCTCTGGA-3' and reverse, 5'-TTTCATTGTCAACTG GGCACT-3'; GAPDH forward, 5'-CTTTGGTATCGTGGA AGGACTC-3' and reverse, 5'-GTAGAGGCAGGGATGATG TTCT-3'.

Western blot analysis. Cells were dissolved by using radioimmunoprecipitation assay buffer (Beyotime Institute of Biotechnology, Haimen, China) and a bicinchoninic acid protein assay kit (cat no. 23225; Thermo Fisher Scientific, Inc.) was used for determining the protein concentration. Protein samples ( $25 \mu \mathrm{g}$ per lane) were separated by $10 \%$ SDS-PAGE, transferred to a polyvinylidene difluoride membrane and blocked with 5\% skimmed milk powder. The membrane was incubated with a primary antibody against GAPDH (cat no. 5174), nuclear factor (NF)-kB (p65; cat no. 8214) or TRAF6 (cat no. 8028) (dilution for all, 1:1,000; Cell Signaling Technology, Inc., Danvers, MA, USA) overnight at $4^{\circ} \mathrm{C}$, and then incubated with anti-rabbit IgG horseradish peroxidase-conjugated secondary antibody (cat no. 7074; 1:5,000 dilution; Cell Signaling Technology, Inc.) at room temperature for $2 \mathrm{~h}$. The protein levels were detected by enhanced chemiluminescence using the Chemiluminescent ECL reagent (EMD Millipore, Billerica, MA, USA).

ELISA. Pro-inflammatory cytokines [interleukin (IL)-6, cat no. E-EL-H0102c; Elabscience Biotechnology, Inc., Wuhan, China), IL-2 (cat no. 8629), TNF- $\alpha$ (cat no. 8668) (both from Cell Signaling Technology, Inc.) and transforming growth factor (TGF)- $\beta$ (cat no. BMS249-4FIVE; Invitrogen; Thermo Fisher Scientific, Inc.) secreted from THP-1-derived macrophages transfected with miR-146b-5p inhibitor or control, followed by treatment with oxidized low-density lipoprotein $(50 \mu \mathrm{g} / \mathrm{ml})$ for $24 \mathrm{~h}$, were detected by using respective ELISA kits according to the manufacturer's instructions.

Luciferase reporter assay. To predict the potential targets of miR-146b-5p, TargetScan (http://www.targetscan. org/vert_71/) was performed in the present study. To confirm our prediction, miR-146b-5p recognition sequences from the wild-type (WT) and mutant (MUT) 3'-UTR of TRAF6 (forward, 5'-GCGATCGCTATATGTAATATATTAAAAGTG AAA-3' and reverse, 5'-GGAGCTCAAATAATTAAGGTT ATATTTAGG-3') were amplified and then cloned into the 


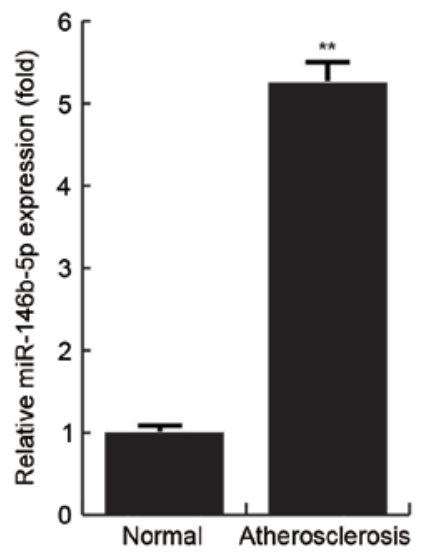

B

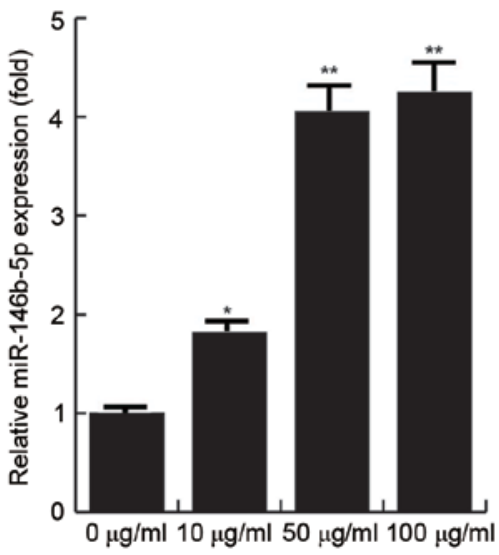

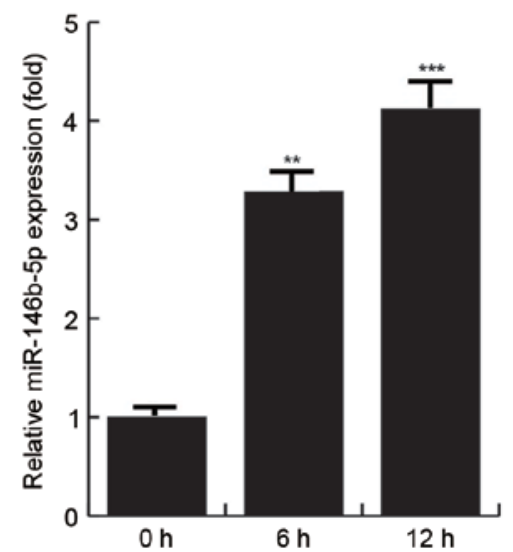

Figure 1. Expression of miR-146b-5p is increased in human macrophages in clinical specimens and is induced by oxLDL. (A) Levels of miR-146b-5p in atherosclerotic lesions $(n=10)$ and normal veins $(n=10)$ from the same patients with AS. (B) Analysis of miR-146b-5p expression in THP-1 cells, which were first stimulated with PMA $(100 \mathrm{nM})$ to induce them to differentiate into macrophages and then treated with oxLDL at the indicated doses $(0,10,50$ or $100 \mu \mathrm{g} / \mathrm{ml}$ ). (C) miR-146b-5p expression in THP-1 cells, which were first stimulated with PMA (100 nM) to induce them to differentiate into macrophages and then treated with oxLDL $(50 \mu \mathrm{g} / \mathrm{ml})$ for the indicated times $(0,6$ or $12 \mathrm{~h})$. miR-146b-5p was determined by reverse-transcription quantitative polymerase chain reaction analysis. ${ }^{*} \mathrm{P}<0.05,{ }^{* *} \mathrm{P}<0.01,{ }^{* * *} \mathrm{P}<0.001$ vs. control. miR, microRNA; oxLDL, oxidized low-density lipoprotein; PMA, phorbol 12 -myristate 13 -acetate.

psiCHECK-2 reporter vector (Promega Corporation, Madison, WI, USA). The resulting miR-146b-5p-TRAF6-3'UTR-WT or miR-146b-5p-TRAF6-3'UTR-MUT vectors were respectively co-transfected with miR-146b-5p or control miR into HEK293T cells using Lipofectamine 2000 transfection reagent in line with the manufacturer's protocol. At $48 \mathrm{~h}$ after transfection, the Dual-Luciferase Reporter Assay system (Promega Corporation) was used for luciferase activity detection. The luciferase activity was then normalized, expressed and Renilla luciferase activity was used as the internal control.

Statistical analysis. Data are presented as the mean \pm standard deviation. Student's t-test was performed for comparison between two groups. SPSS 16.0 software (SPSS, Inc., Chicago, IL, USA) was used for statistical analysis. All tests were independently performed at least three times. $\mathrm{P}<0.05$ was considered to indicate a statistically significant difference.

\section{Results}

miR-146b-5p is overexpressed in the atherosclerotic lesions of patients with $A S$ and is induced by oxLDL in human macrophages. The relative expression levels of miR146b-5p in atherosclerotic lesions and normal veins from the same AS patients were detected by RT-qPCR, and the results demonstrated that the miR146b-5p expression levels were significantly increased in the atherosclerotic lesions compared with those in the normal veins. To determine whether overexpression of miR-146b-5p is induced by oxLDL, THP-1 cells were first treated with $100 \mathrm{nM}$ PMA to stimulate their differentiation into macrophages and then stimulated with various concentrations of oxLDL $(0,10,50$ and $100 \mu \mathrm{g} / \mathrm{ml})$ for $24 \mathrm{~h}$ or with $50 \mu \mathrm{g} / \mathrm{ml}$ oxLDL for specific durations $(0,6$ or $12 \mathrm{~h})$ in order to induce foam cell formation (18). The findings demonstrated that the expression of miR-146b-5p was significantly increased by oxLDL stimulation, and the effect was dose- and time-dependent (Fig. 1).

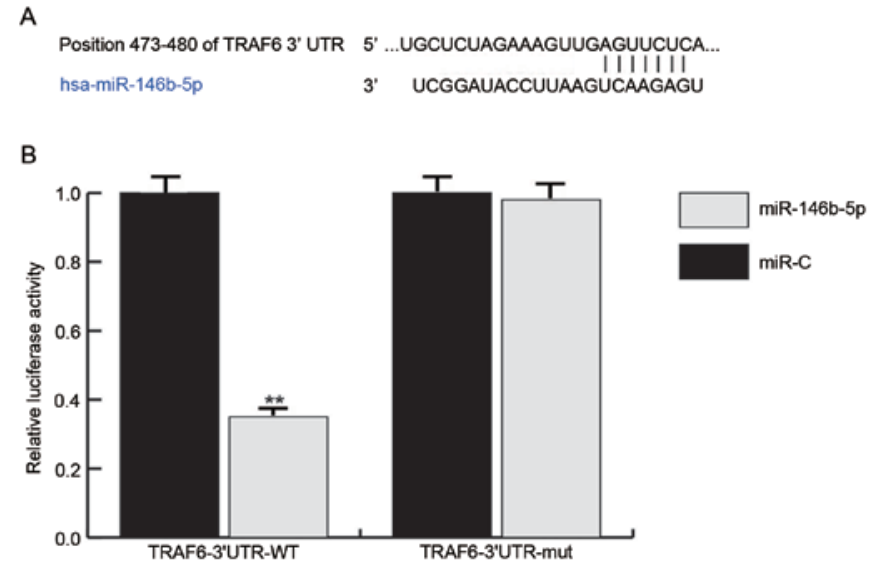

Figure 2. miR-146b-5p directly targets TRAF6. (A) Interaction between miR-146b-5p and the 3'UTR of TRAF6 was predicted using TargetScan and MiRanda databases. (B) Luciferase activity of cells transfected with a reporter vector containing a WT or mutant TRAF6 3'UTR together with miR-146b-5p or miR-C are presented in the bar graph. ${ }^{* *} \mathrm{P}<0.01$ vs. control. TRAF6 3'UTR-MUT, TRAF6 3'UTR with a mutation in the miR-146b-5p binding site; UTR, untranslated region; WT, wild-type; TRAF6, tumor necrosis factor receptor-associated factor 6 ; $\mathrm{miR}$, microRNA; miR-C, control miR; hsa, Homo sapiens.

TRAF6 is a functional target of miR-146b-5p. To explore the mechanisms by which miR-146b-5p affects macrophage-derived foam cell formation and the inflammatory response, TargetScan and miRanda database searches were performed to predict the direct mRNA targets of miR-146b-5p. To confirm the prediction of TRAF6 mRNA being a target, a luciferase reporter assay was performed. The results revealed that the luciferase activity was significantly decreased in the HEK293 cells co-transfected with miR-146b-5p and miR-146b-5p-TRAF6-WT, while co-transfection of miR-146b-5p with miR-146b-5p-TRAF6-MUT did not (Fig. 2). This result indicated that TRAF6 is a direct target of miR-146b-5p. 
A

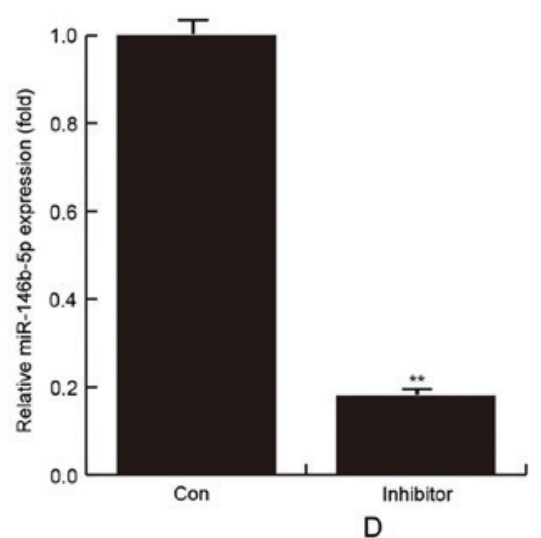

B

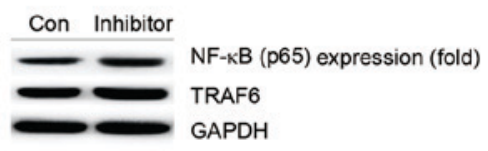

C

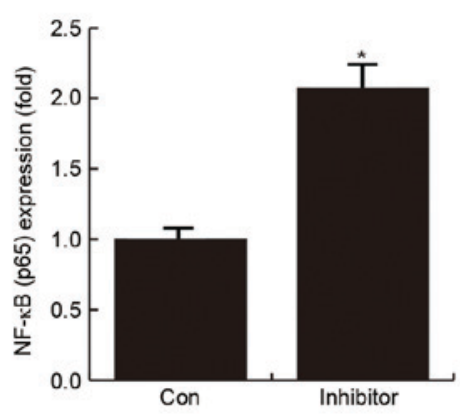

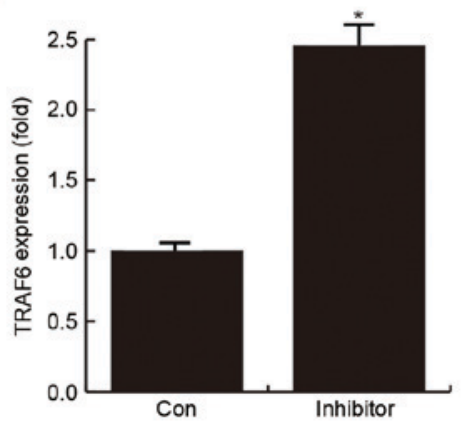

E

Figure 3. Confirmation of miR-146-5p inhibition and its effect on the protein levels of TRAF6 and NF- $\mathrm{kB}$ (p65) as well as TRAF6 mRNA expression. (A) Relative miR-146-5p level; (B) protein level of TRAF6 and NF- $\mathrm{kB}$ (p65); protein expression levels of (C) NF- $\mathrm{kB}$ (p65) and (D) TRAF6 were expressed as fold changes compared with the control group; and (E) Relative TRAF6 mRNA expression. THP-1 cells, which had been treated with $100 \mathrm{nM}$ phorbol 12-myristate 13-acetate for $12 \mathrm{~h}$ to differentiate them into macrophages, were transfected with miR-146b-5p inhibitor or its negative control for $24 \mathrm{~h}$, and then treated with $50 \mu \mathrm{g} / \mathrm{ml}$ oxidized low-density lipoprotein for $24 \mathrm{~h}$. RT-qPCR and western blot analysis were then used for miRNA/mRNA and protein detection. "P<0.05, " $\mathrm{P}<0.01$ vs. control. TRAF6, tumor necrosis factor receptor-associated factor 6; miR, microRNA; Con, control; NF, nuclear factor; RT-qPCR, reverse-transcription quantitative polymerase chain reaction.

miR-146b-5p inhibition during foam cell formation increases TRAF6. To investigate the role of miR-146b-5p in the pathological process of AS, miR-146b-5p was inhibited using miR-146b-5p inhibitor. THP-1 cells, which had been treated with $100 \mathrm{nM}$ PMA for $12 \mathrm{~h}$ to induce their differentiation into macrophages, were transfected with miR-146b-5p inhibitor or its negative control for $24 \mathrm{~h}$, and then treated with $50 \mu \mathrm{g} / \mathrm{ml}$ oxLDL for 24 hto induce foam cell formation. Efficient inhibition of miR-146b-5p was confirmed by RT-qPCR (Fig. 3A).

In addition, western blot analysis demonstrated that after transfection with miR-146b-5p inhibitor for $24 \mathrm{~h}$, the protein levels of $\mathrm{NF}-\kappa \mathrm{B}(\mathrm{p} 65)(\mathrm{P}<0.05$; Fig. 3B and $\mathrm{C})$ and TRAF6 were significantly enhanced ( $\mathrm{P}<0.05$; Fig. $3 \mathrm{~B}$ and $\mathrm{D})$. The results also indicated that miR-146b-5p inhibition may promote the inflammatory response, possibly by promoting the expression of $N F-\kappa B(p 65)$ in foam cell formation. At the mRNA level, TRAF6 was significantly enhanced in the group transfected with miR-146b-5p inhibitor (Fig. 3E). This further supported the result that TRAF6 is a direct target of miR-146b-5p.

miR-146b-5p inhibition promotes the inflammatory response during foam cell formation. The effect of miR-146b-5p inhibition on inflammatory factor secretion in macrophages treated with oxidized low-density lipoprotein $(50 \mu \mathrm{g} / \mathrm{ml})$ for $24 \mathrm{~h}$ was examined by ELISA. The results demonstrated that in the group transfected with miR-146b-5p inhibitor, the secretion of IL-6, IL-2, TNF- $\alpha$ and TGF- $\beta$ was significantly increased (Fig. 4).

miR-146b-5p inhibition enhances lipid uptake during foam cell formation. The effect of miR-146b-5p inhibition on foam cell formation was analyzed by Oil Red $\mathrm{O}$ staining. The results indicated that in the group transfected with miR-146b-5p, lipid uptake was notably enhanced (Fig. 5).

\section{Discussion}

Increasing evidence suggested that chronic inflammation contributes to the formation of atherosclerotic lesions. Foam cell formation may be induced by exposure of macrophages to oxLDL (19). Exploration of the potential molecular mechanisms of inflammatory processes and foam cell formation will provide novel strategies for the treatment of AS.

Various studies have demonstrated that miRNAs have an important role in the pathogenesis of AS. Zernecke et al (20) demonstrated that miR-126 prevents atherosclerotic lesion formation through regulating angiogenesis and vascular inflammation, and an anti-AS function of miR-126-5p has been reported recently (21). Consistent with these results, Tabet et al (22) suggested that the anti-inflammatory function of high-density lipoprotein is conferred through miR-223. By contrast, Zhang et al (23) reported that miR-150 promotes 

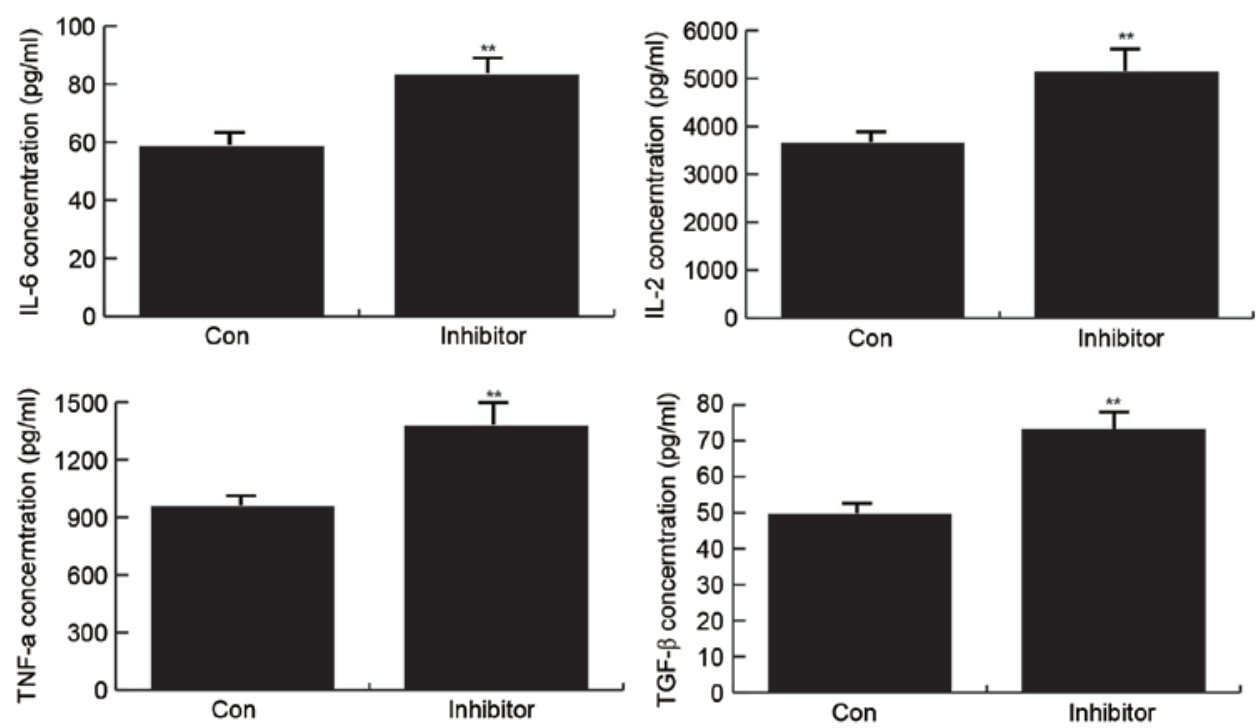

Figure 4. miR-146b-5p inhibition promotes inflammation. After transfection of miR-146-5p inhibitor or its negative control into macrophages, the cells were treated with oxidized low-density lipoprotein $(50 \mu \mathrm{g} / \mathrm{ml})$ for $24 \mathrm{~h}$, and the secreted protein concentration of IL-6, IL-2, TNF- $\alpha$ and TGF- $\beta$ was determined by ELISA. Values are expressed as the mean \pm standard deviation of 3 independent experiments as determined by Student's t-test. ${ }^{* *} \mathrm{P}<0.001$ vs. Con. IL, interleukin; TNF, tumor necrosis factor; TGF, transforming growth factor; miR, microRNA; Con, control.

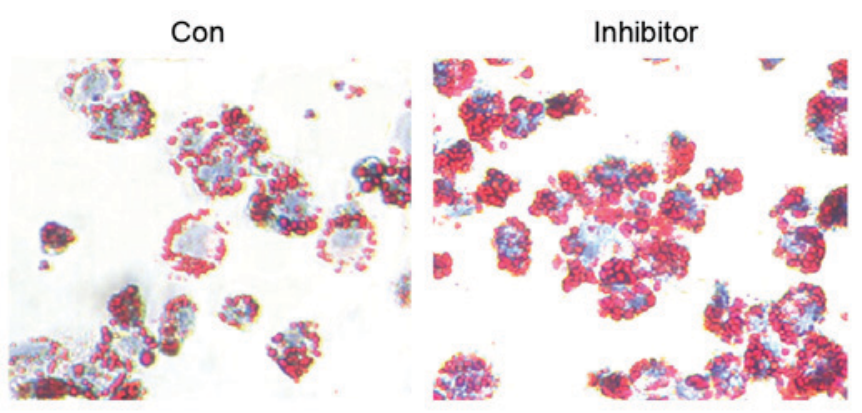

Figure 5. miR-146b-5p inhibition enhances lipid uptake. Lipid uptake was measured by Oil Red O staining after transfection of miR-146b-5p inhibitor or its negative control into macrophages, followed by treatment with oxidized low-density lipoprotein $(50 \mu \mathrm{g} / \mathrm{ml})$ for $24 \mathrm{~h}$ (magnification, $\mathrm{x} 40)$. miR, microRNA; Con, control.

AS via enhancing endothelial cell migration. The present study investigated the role of miR-146b-5p in AS-associated processes to identify novel therapeutic strategies for the treatment of AS and other vascular diseases.

First, the expression levels of miR-146b-5p were determined in atherosclerotic lesions of patients with AS, and the results indicated that miR-146b-5p was highly expressed in the atherosclerotic lesions compared with that in normal veins from the same patient. The present study also found that miR-146b-5p may be induced by oxLDL stimulation in a time- and dose-dependent manner, which was consistent with the results of a previous study (13).

Next, the present study confirmed TRAF6 as a direct target of miR-146b-5p. A statistically significant inverse association was identified between miR-146b-5p and TRAF6 expression in oxLDL-stimulated macrophages, suggesting a significant biological function of the TRAF6-miR-146b-5p complex in AS. TRAF6 functions as a signal transducer in the $N F-\kappa B$ pathway, and the activation of $N F-\kappa B$ was reported to be elevated in patients with acute coronary syndrome and in oxLDL-induced mast cells $(24,25)$. Various molecules involved in the immune response and early inflammation are modulated by the NF- $\kappa \mathrm{B}$ pathway. Thus, it was hypothesized that TRAF6-miR-146b-5p is involved in the regulation of AS-associated inflammation. The results of the present study suggested that inhibition of miR146b-5p increases TRAF6 and $\mathrm{NF}-\kappa \mathrm{B}(\mathrm{p} 65)$ expression, as well as the secretion of pro-inflammatory cytokines (IL-6, IL-2, TNF- $\alpha$ and TGF- $\beta$ ) in oxLDL-simulated macrophages. These results confirmed the prediction that miR-146b-5p acts as a promoter of inflammation in oxLDL-stimulated macrophages, partly via targeting TRAF6. Furthermore, the present study investigated the effect of miR-146b-5p on foam cell formation via Oil Red $\mathrm{O}$ staining. The results indicated that miR-146b-5p inhibition significantly enhanced the lipid uptake by oxLDL-stimulated macrophages.

In conclusion, the present study found that miR146b-5p is overexpressed in the atherosclerotic lesions of patients with AS, and it may be induced by oxLDL in human macrophages. Blockade of miR146b-5p promoted inflammation and foam cell formation by increasing TRAF6-mediated activation of $N F-\kappa B$ expression, indicating the anti-AS function of miR146b-5p.

\section{References}

1. Libby P, Ridker PM and Hansson GK: Progress and challenges in translating the biology of atherosclerosis. Nature 473: 317-325, 2011.

2. Hansson GK: Inflammation, atherosclerosis, and coronary artery disease. N Engl J Med 352: 1685-1695, 2005.

3. Imanishi T and Akasaka T: Novel strategies to target inflammatory processes in atherosclerosis. Curr Pharm Des 19: 1616-1625, 2013.

4. Moore KJ and Tabas I: Macrophages in the pathogenesis of atherosclerosis. Cell 145: 341-355, 2011.

5. Zhang C: MicroRNAs in vascular biology and vascular disease. J Cardiovasc Transl Res 3: 235-240, 2010.

6. Zhang C: MicroRNAs: Role in cardiovascular biology and disease. Clin Sci (Lond) 114: 699-706, 2008. 
7. Ambros V: microRNAs: Tiny regulators with great potential. Cell 107: 823-826, 2001.

8. Eulalio A, Huntzinger E and Izaurralde E: Getting to the root of miRNA-mediated gene silencing. Cell 132: 9-14, 2008.

9. Li X, Kong D, Chen H, Liu S, Hu H, Wu T, Wang J, Chen W, Ning Y, Li Y and Lu Z: miR-155 acts as an anti-inflammatory factor in atherosclerosis-associated foam cell formation by repressing calcium-regulated heat stable protein 1 . Sci Rep 6: 21789, 2016.

10. Xu Z, Han Y, Liu J, Jiang F, Hu H, Wang Y, Liu Q, Gong Y and Li X: miR-135b-5p and miR-499a-3p promote cell proliferation and migration in atherosclerosis by directly targeting MEF2C. Sci Rep 5: 12276, 2015.

11. Zhang Y, Qin W, Zhang L, Wu X, Du N, Hu Y, Li X, Shen N, Xiao D, Zhang H, et al: MicroRNA-26a prevents endothelial cell apoptosis by directly targeting TRPC6 in the setting of atherosclerosis. Sci Rep 5: 9401, 2015.

12. Ouimet M, Ediriweera HN, Gundra UM, Sheedy FJ, Ramkhelawon B, Hutchison SB, Rinehold K, van Solingen C, Fullerton MD, Cecchini K, et al: MicroRNA-33-dependent regulation of macrophage metabolism directs immune cell polarization in atherosclerosis. J Clin Invest 125: 4334-4348, 2015.

13. Chen T, Huang Z, Wang L, Wang Y, Wu F, Meng S and Wang C: MicroRNA-125a-5p partly regulates the inflammatory response, lipid uptake, and ORP9 expression in oxLDL-stimulated monocyte/macrophages. Cardiovasc Res 83: 131-139, 2009.

14. Zhu J, Chen T, Yang L, Li Z, Wong MM, Zheng X, Pan X, Zhang L and Yan H: Regulation of microRNA-155 in atherosclerotic inflammatory responses by targeting MAP3K10. PLoS One 7: e46551, 2012.

15. Daigneault M, Preston JA, Marriott HM, Whyte MK and Dockrell DH: The identifcation of markers of macrophage differentiation in PMA-stimulated THP-1 cells and monocyte-derived macrophages. PLoS One 5: e8668, 2010.

16. Bao Y, Wang L, Xu Y, Yang Y, Wang L, Si S, Cho S and Hong B: Salvianolic acid B inhibits macrophage uptake of modifed low density lipoprotein (mLDL) in a scavenger receptor CD36-dependent manner. Atherosclerosis 223: 152-159, 2012.
17. Livak KJ and Schmittgen TD: Analysis of relative gene expression data using real-time quantitative PCR and the 2(-Delta Delta C(T)) method. Methods 25: 402-408, 2001.

18. Zhu GF, Yang LX, Guo RW, Liu H, Shi YK, Wang H, Ye JS, Yang ZH and Liang X: miR-155 inhibits oxidized low-density lipoprotein-induced apoptosis of RAW264.7 cells. Mol Cell Biochem 382: 253-261, 2013.

19. Weber C and Noels H: Atherosclerosis: Current pathogenesis and therapeutic options. Nat Med 17: 1410-1422, 2011.

20. Zernecke A, Bidzhekov K, Noels H, Shagdarsuren E, Gan L, Denecke B, Hristov M, Köppel T, Jahantigh MN, Lutgens E, et al: Delivery of microRNA-126 by apoptotic bodies induces CXCL12-dependent vascular protection. Sci Signal 2: ra81, 2009.

21. Schober A, Nazari-Jahantigh M, Wei Y, Bidzhekov K, Gremse F, Grommes J, Megens RT, Heyll K, Noels H, Hristov M, et al: MicroRNA-126-5p promotes endothelial proliferation and limits atherosclerosis by suppressing Dlk1. Nat Med 20: 368-376, 2014.

22. Tabet F, Vickers KC, Cuesta Torres LF, Wiese CB, Shoucri BM, Lambert G, Catherinet C, Prado-Lourenco L, Levin MG, Thacker S, et al: HDL-transferred microRNA-223 regulates ICAM-1 expression in endothelial cells. Nat Commun 5: 3292, 2014.

23. Zhang Y, Liu D, Chen X, Li J, Li L, Bian Z, Sun F, Lu J, Yin Y, Cai X, et al: Secreted monocytic miR-150 enhances targeted endothelial cell migration. Mol Cell 39: 133-144, 2010.

24. Fang H, Lin J, Wang L, Xie P, Wang X, Fu J, Ai W, Chen S, Chen F, Zhang F, et al: Kruppel-like factor 2 regulates dendritic cell activation in patients with acute coronary syndrome. Cell Physiol Biochem 32: 931-941, 2013.

25. Meng Z, Yan C, Deng Q, Dong X, Duan ZM, Gao DF and Niu XL: Oxidized low-density lipoprotein induces inflammatory responses in cultured human mast cells via Toll-like receptor 4. Cell Physiol Biochem 31: 842-853, 2013. 